Volume 8, No.1.6, 2019

International Journal of Advanced Trends in Computer Science and Engineering

Available Online at http://www.warse.org/IJATCSE/static/pdf/file/ijatcse0881.62019.pdf

https://doi.org/10.30534/ịatcse/2019/0881.62019

\title{
Efficiency of Similarity Measures in Content based Retrieval Using Texture, Shape and Color
}

\author{
Poornima Raikar ${ }^{1}$, Dr. S.M Joshi ${ }^{2}$ \\ ${ }^{1}$ VDIT,Haliyal-581329 , Karnataka, India \\ 1pnraikar10@gmail.com \\ ${ }^{2}$ SDMCET, Dharwad -580002, Karnataka, India \\ joshshree@yahoo.com
}

\begin{abstract}
Content based image retrieval (CBIR) provides efficient way to retrieve the images from the databases. The feature extraction and similar attribute measures are the key factors for retrieval performance. We need efficient way to access the visual content from large database. Content based image retrieval (CBIR) provides the solution for efficient retrieval of images from large image database. In this work hybrid feature based CBIR system is proposed with comparison of various distance measures. Spatial features like color histogram, color auto-correlogram, color moments, HSV histogram features and Frequency domain features like Semantic image features Gabor wavelet mean entropy, amplitude, energy. Wavelet moments like mean and the standard deviation of the transform coefficients, Shape feature histogram of oriented gradient, Hu moments are used to form the feature vector. The experiments are performed on flower database which consists of 1360 images from 17 different classes. For our experiment we have chosen 3 different classes of flowers of same color consisting of 100 images each. Experimental result shows that the proposed approach performs better in terms of precision, recall, accuracy of classifier and similarity measures. Shape feature play an prominent role for images with same color, texture. We made the comparison of efficiency of different similarity measures like Mahalanobis , Euclidean, Correlation, Spearman, City block(Manhattan) distances approaches on different images based on color, texture, shape features and found which distance measure is best based on performance.
\end{abstract}

Key words: HOG, SVM, Confusion matrix, Hu moments

\section{INTRODUCTION}

In today's world, knowledge is augmenting day by day, the most enriched research area in digital image processing is image retrieval system. With increasing number of images accessible through internet every day and stored in databanks, Need for effective and scalable search algorithms has become an important research area. Content Based Image Retrieval (CBIR) is the method of searching similar images from the database based on their visual contents and has applications in many areas. There are various challenges which affect the designing of a CBIR system like dimension of feature vector, image features selection, retrieval algorithm and presentation method for final results [3].Difficult is to fill the semantic gap by using only low level features or only high level features of an image because of the large diversity of image databases. Retrieval algorithm and method for presenting final results need to be improved so that image features can better represent the semantics of the images where we need to have fusion of image features. Objective is to retrieve query from a large collection of database we develop a CBIR system capable of retrieval based on feature such as color, texture, shape for flower image given below in Figure 1

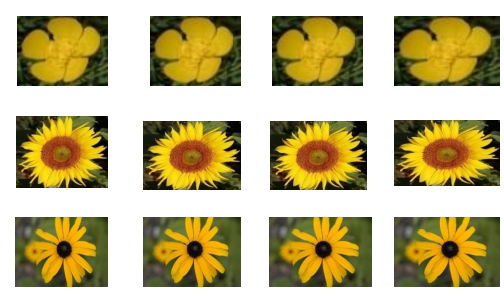

Figure 1: Images stored in the database with 3 classes of flowers. (a) sunflower (b) buttercup (c) coneflower

We highlight on the feature extraction, features based matching and indexing method which represents the base of recall images. In this paper we discuss about how to retrieve the images using shape, texture and color descriptor method for retrieval, then experimentation results show how shape feature is important feature when same images are considered and also present comparison of efficiency of similarity measures.

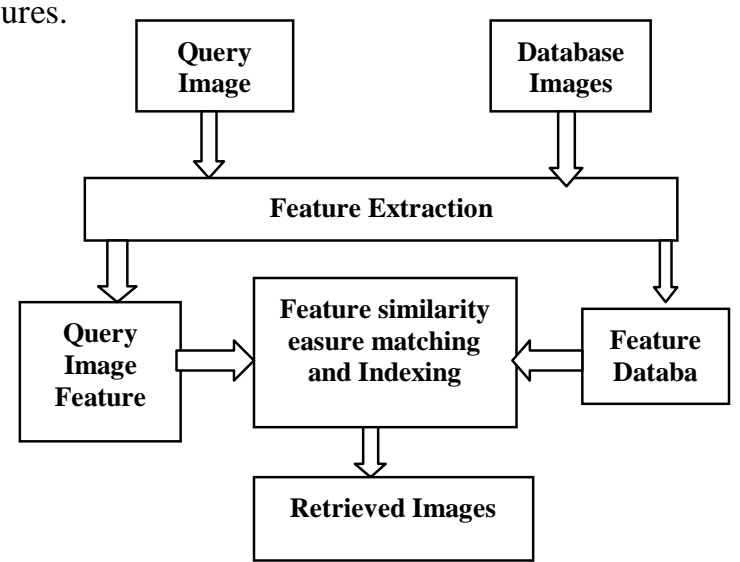

Figure 2: Diagram depicting content based Image Retrieval 
The steps involved in content based image retrieval are shown above in Figure 2. Image Database contains set of training images in the database. First we extract features of the images stored in the database then store in feature database, Query image feature are extracted and are compared with image features of database images, then image features are indexed and matched, whichever images are matched to the query images are retrieved as output.

\section{LITERATURE SURVEY}

CBIR has come long way since 1990 till date. There are several retrieval algorithms that process image into several layers of tasks. These tasks consist of extracting the multidimensional features of an image query and compare it with images in the database. Database with extracted information from the images and indexed appropriately will affect the performance of retrieval.. We compare the features such as spatial and frequency features by considering five types of Distance Metric. Ruigang Fu, Biao Li, Yinghui Gao, Ping Wang[1] proposed deep features generated by convolution neural network (CNN) to CBIR, and uses linear support vector machine (SVM) to train a hyperplane which can separate similar and dissimilar image pairs where to extract the feature representations $\mathrm{CNN}$ used and to learn the similarity measures. Mayank Jain and Divakar Singh[2] In this paper, author proposed the CBIR technique which used Color features like color moments, color histogram, invariant color histogram, and dominant color.. Color based CBIR technique has some limitations. So to enhance the color based method uses texture features like Gray Level Co occurrence matrix (GLCM), Gabor Transform and Tamura Features and Shape features like Histogram of Edge Directions, Region Moments, invariant Moments, Zernike moments, Legendre Moments. Proposed CBIR systems provide maximum support in bringing the semantic gap between the simplicity of available visual feature and the richness of the semantics. Ramesh K Lingadalli,N.Ramesh[3] proposed that single feature represent only part of the image property to improve the image retrieval we must make use of multiple features such as HSV color, approximate shape and texture to represent the whole image property. Satish Tunga, D.Jayadevappa \& C.Gururaj[4] made comparison of various approaches of CBIR and reviewed available algorithms. In this paper they presented comparative results of various techniques and discussed advantages, disadvantages and limitations. They proposed methodologies used for extracting low level features and various distance measures to find the similarity between images in reducing the semantic.gap. Nishant Shrivastava, Vipin Tyagi [5] author analyze images using different learning algorithms for image retrieval. The color and shape based image descriptors are used to distinguish specific set of images from the whole database. Support Vector Machine (SVM), K-Nearest Neighbour (KNN), Naïve-Bayes and Random Forest are trained by this feature vectors are used for class prediction. SVM and Naïve-Bayes classification technique on the combined feature set provides the best results..Yogita Mistry, D.T. Ingole, M.D. Ingole[6] Author has proposed hybrid CBIR system using a various distance measure. Spatial domain features includes color features like auto-correlogram, color moments, HSV histogram features ,frequency domain features like moments using SWT, texture features using Gabor wavelet transform are used. To enhance precision binarized statistical image features, color and edge directivity descriptor features are employed for developing efficient CBIR system. . The experiments are performed using WANG database which consists of 1000 images from 10 different classes. Experimental result shows that the proposed approach performs better in terms of precision compared to other existing systems. Wengang Zhou, Houqiang $\mathrm{Li}$, and Qi Tian[9] proposed Content-based image retrieval (CBIR) system, that makes use of the representation of visual content to identify relevant images. The purpose of this paper is to categorize and evaluate those algorithms proposed during the period of 2003 to 2016. D. Sarala1, Tejas Kanikdaley, Sharad Jogi ,Rahul K. Chaurasiya[12] proposed method that combines both color and texture features of an image for image retrieval in a hierarchical manner and shows its advantage. This paper also introduces an effective method of image segmentation for feature extraction. The proposed hierarchical approach was applied to the standard INRIA dataset. A significant improvement in the recall and precision is achieved in this proposed method .G. Bindu Madhavi, V. Vijaya Kumar, K. Sasidhar[14] proposed new framework for content based image retrieval (CBIR) by deriving color features and texture features This paper initially transforms the RGB color plane image into HSV color plane and derives the individual histograms on $\mathrm{H}, \mathrm{S}$ and $\mathrm{V}$ planes..

\section{PROPOSED WORK}

\subsection{Methodology}

Shape is an important visual feature and describes geometric feature .Whenever we consider images of same colors like sunflower, buttercup, coneflower, dandelion etc color, texture of images will not be sufficient in this case shape feature plays a most leading role. Representation and description of shape is a difficult task Shape descriptor can been contour or region based can be used to describe shape like histogram of gradient, boundary, edge, area, perimeter, convex hull etc. In this work we consider Histogram of oriented gradient, $\mathrm{Hu}$ moments for shape feature representation, Spatial features like Color Features like Color histogram HSV space is chosen, each $\mathrm{H}, \mathrm{S}, \mathrm{V}$ component is uniformly quantized into 8,2 and 2 bins respectively. Color auto-correlogram where the image is quantized into $4 \times 4 \times 4$ colors in the RGB space. Color moments like mean and standard deviation from the R, G, B color channels are extracted. Frequency domain like semantic features like Gabor wavelet filters spanning four scales and six orientations are used. Wavelet moments used to apply wavelet transform to the image with a 4-level decomposition, the mean and the standard deviation of the transform coefficients are used to form the feature vector. The output is a vector of the features values. 


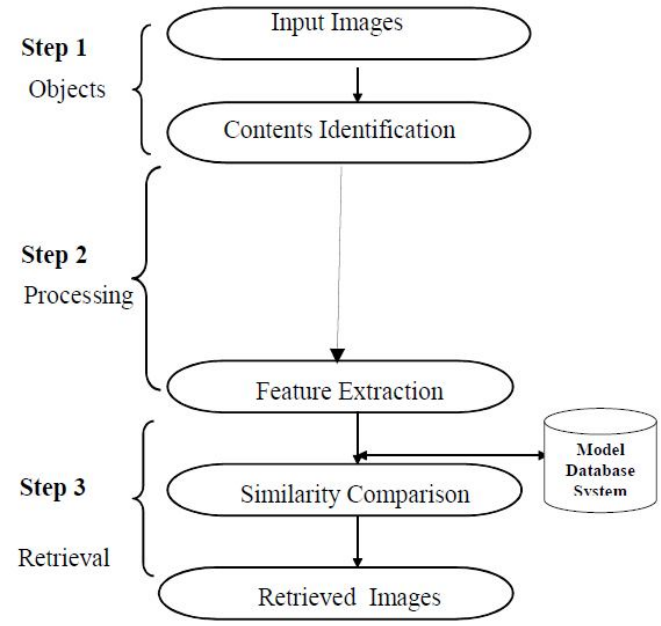

Figure 3: Steps for proposed algorithm

The basic steps involved in the proposed retrieval system include database normalization, processing, creation of feature database, comparison and image retrieval as shown in Fig 3. Steps of the proposed algorithm are as follows.

1. Input: A Query image[IMAGE ACQUSITION]

2. Pre-processing[ removal of noise, smoothing]

3. Pre-processed Image

4. Feature extraction[Color, shape, texture feature]

5. Indexing and Similarity comparisons between input image and Database[matching and retrieval]

5. Output Images

We focus on both global features and local features. Global features represent interest points and takes entire image globally. Local features represents limited regions of an image in which interest points are determined in the entire image and neighbouring image points .Here the dataset includes 300 images of 3 classes like buttercup, sunflower, coneflowers 100 each images. Similarity measures helps us to match the similarity between these two feature extraction. Then the matched images are retrieved.

\section{SIMILARITY MEASUREMENTS}

Image retrieval calculates visual similarities between a query image and images in a database. Different similarity distance measures will affect retrieval performances of an image significantly[6].

The different distance measures used for matching are as follows

1. Mahalanobis Distance

2. Euclidean Distance(L2)

3. Correlation Distance

4. Spearman Distance

5. City block distance

\subsection{Mahalanobis Distance}

Mahalanobis distance is a measure of the distance between point $P$ and distributionCalculates similarity between random multidimensional D. It is multivariate distance .

$$
d=\sqrt{(\bar{x})}-(\mu)^{T} S^{-1}(\bar{x})-(\mu)
$$

\subsection{Euclidean Distance}

Euclidean distance is called as L2 method. This similarity measure is distance between points or straight line on Cartesian coordinate system. This method measures the files distance.

$$
d=\sqrt{\sum\left|x_{i}-y_{i}\right|^{2}}
$$

Deriving the Euclidean distance between two data points involves computing the square root of the sum of the squares of the differences between corresponding values.

\subsection{Correlation Distance}

Similarity measure is distance between two sets of correspondence. For database $\mathrm{N}, \mathrm{N}$ means features and $\mathrm{N}^{\prime}=\mathrm{N}(\mathrm{N}-1) / 2$ image pairs. To detect the correlation we create distance matrix. Here in the below formula $\mathrm{N}$ is a database, $\mathrm{M}$ means features and $\mathrm{N}^{\prime}=\mathrm{N}(\mathrm{N}-1) / 2$ image pairs.

$$
\begin{gathered}
\sum m m i=\frac{1}{N} \sum_{n=1}^{N} D_{n m} D_{n m i}\left(\frac{1}{N} \sum_{n=1}^{N} D_{n m}\right)\left(\frac{1}{N} \sum_{n=1}^{\mid N} D_{n m}\right) \\
R_{m m}=\frac{\sum m m}{\sqrt{\sum m m} \sum m m}
\end{gathered}
$$

$D_{n m}$ and $D_{n m}$ ' are distance of $n^{\text {th }}$ image comparison using $m$ feature and $\mathrm{m}$ ' feature.

\subsection{Spearman distance}

It shows both the basic histogram intersection method and size weighed histogram are very close to similarity. Spearman correlation coefficient is 0.915 . Measure of rank correlation coefficient two points

$$
D(x, y)=\frac{6 \sum_{j=1}^{d}\left[R\left(x_{i}\right)-R\left(y_{j}\right)\right]^{2}}{d\left(d^{2}-1\right)}
$$

\subsection{City block Distance}

City block distance is the distance of each pixel between 2 coordinates. For example distance between 2 adjacent pixel is 1 because they are adjacent. This is used for edge detection applications as shown in Fig 4

$$
d=\sum_{i=1}^{n}\left|x_{i}-y_{i}\right|
$$

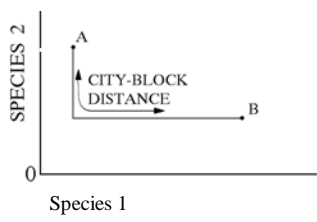

Figure 4: edge detection good result but for frangipani flower all four distance measure gave good results.. Precision indicates that an algorithm returned substantially more relevant results than irrelevant ones, while high recall means that an algorithm returned most of the relevant results[6].

\section{COMPARISON OF EFFICIENCY OF METHOD}

Here the dataset includes different classes like buttercup, sunflower, Coneflower each containing 100 images 300 samples divided into 50\% training and 50\% testing samples. 
Support vector machine is used as classifier. Confusion matrix shows the comparison of correctly and incorrectly retrieved images, diagonal represents correctly classified and non diagonal represents incorrectly classified data based on precision and recall. Comparison of methods are recorded in Table 1,Table 2,Table 3 for each flower class are given below. Bar Graph of precision ,recall, accuracy of classifier ,error rate for each flower class based on different similarity measures are given below in Fig 5,Fig 6,Fig 7 respectively with respect to number of returned images with number of correctly retrieved images diagonally. Confusion matrix used for calculating precision and recall given the predicted labels from a model.

Table 1 : Comparison of efficiency for buttercup

\begin{tabular}{|l|l|c|c|c|c|c|}
\hline $\begin{array}{l}\text { S1 } \\
\text { No }\end{array}$ & $\begin{array}{l}\text { Method } \\
\text { image }\end{array}$ & Precision & Recall & Accuracy & $\begin{array}{l}\text { Error } \\
\text { rate }\end{array}$ \\
\hline 1 & $\begin{array}{l}\text { MAHALANOBIS } \\
\text { DISTANCE }\end{array}$ & f & 72.5 & 90 & 84 & 10 \\
\hline 2 & $\begin{array}{l}\text { EUCLEDIAN } \\
\text { DISTANCE (L2) }\end{array}$ & $\mathbf{f}$ & 76.6 & 92 & 88 & 8 \\
\hline 3 & $\begin{array}{l}\text { CORRELATION } \\
\text { DISTANCE }\end{array}$ & $\mathbf{f}$ & 73.4 & 94 & 86 & 6 \\
\hline 4 & $\begin{array}{l}\text { SPEARMAN } \\
\text { DISTANCE }\end{array}$ & $\mathbf{f}$ & 92.5 & 100 & 96.67 & 5 \\
\hline 5 & $\begin{array}{l}\text { CITYBLOCK } \\
\text { DISTANCE }\end{array}$ & $\mathbf{f}$ & 91.6 & 88 & 91.33 & 12 \\
\hline
\end{tabular}

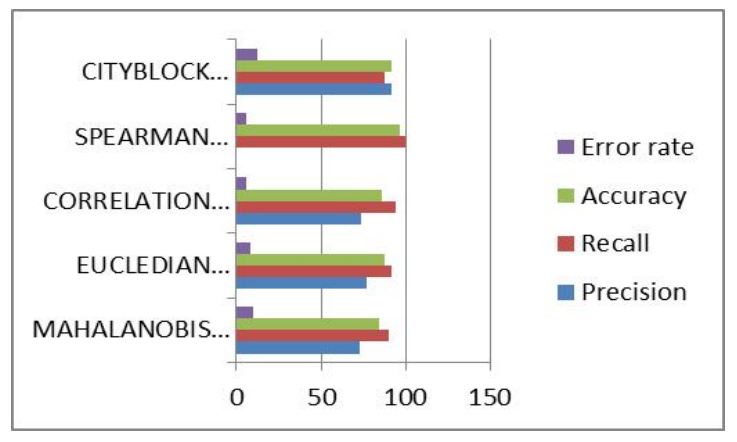

Figure 5:.A bar graph shows comparison against returned images and correctly retrieved images for buttercup

Table 2 :Comparison of efficiency for Sunflower

\begin{tabular}{|l|l|c|c|c|c|c|}
\hline $\begin{array}{l}\text { Sl } \\
\text { No }\end{array}$ & $\begin{array}{l}\text { Query } \\
\text { image }\end{array}$ & Precision & Recall & Accuracy & $\begin{array}{l}\text { Error } \\
\text { rate }\end{array}$ \\
\hline 1 & $\begin{array}{l}\text { MAHALANOBIS } \\
\text { DISTANCE }\end{array}$ & $\begin{array}{l}\text { EUCLEDIAN } \\
\text { DISTANCE (L2) }\end{array}$ & 82.4 & 94 & 90,67 & 6 \\
\hline 3 & $\begin{array}{l}\text { CORRELATION } \\
\text { DISTANCE }\end{array}$ & 81.6 & 80 & 86 & 20 \\
\hline 4 & $\begin{array}{l}\text { SPEARMAN } \\
\text { DISTANCE }\end{array}$ & 88 & 88 & 91.33 & 12 \\
\hline 5 & $\begin{array}{l}\text { CITYBLOCK } \\
\text { DISTANCE }\end{array}$ & 96 & 100 & 95.96 & 5 \\
\hline
\end{tabular}

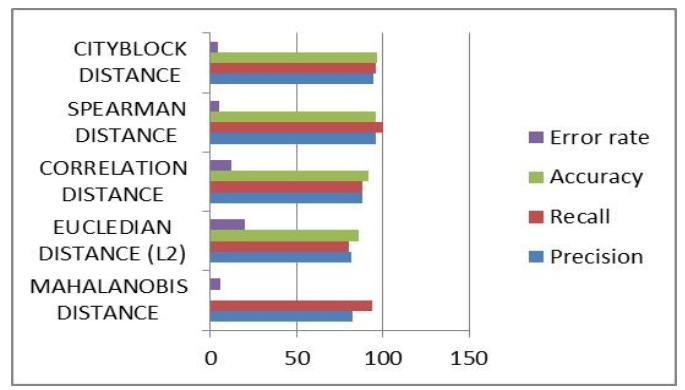

Figure 6:.A bar graph shows comparison against returned image and correctly retrieved images for sunflower

Table 3: Comparison of efficiency for coneflower

\begin{tabular}{|c|l|c|c|c|c|c|}
\hline $\begin{array}{c}\text { Sl. } \\
\text { no }\end{array}$ & \multicolumn{1}{|c|}{ Method } & $\begin{array}{l}\text { Query } \\
\text { image }\end{array}$ & Precision & Recall & Accuracy & $\begin{array}{c}\text { Error } \\
\text { rate }\end{array}$ \\
\hline 1 & $\begin{array}{l}\text { MAHALANOBIS } \\
\text { DISTANCE }\end{array}$ & $\begin{array}{l}\text { EUCLEDIAN } \\
\text { DISTANCE (L2) }\end{array}$ & 90.3 & 94 & 94 & 6 \\
\hline 3 & $\begin{array}{l}\text { CORRELATION } \\
\text { DISTANCE }\end{array}$ & 82.3 & 84 & 87.33 & 16 \\
\hline 4 & $\begin{array}{l}\text { SPEARMAN } \\
\text { DISTANCE }\end{array}$ & 80 & 96 & 90 & 4 \\
\hline 5 & $\begin{array}{l}\text { CITYBLOCK } \\
\text { DISTANCE }\end{array}$ & 85 & 94 & 92.67 & 6 \\
\hline
\end{tabular}

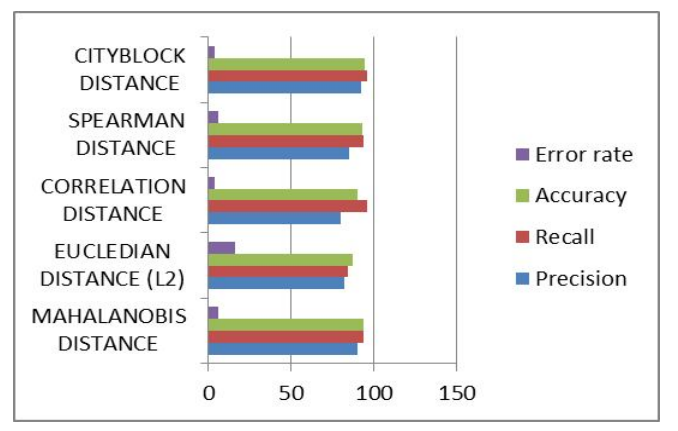

Figure 7 :A bar graph shows comparison against returned images and correctly retrieved images for Coneflower

\section{EXPERIMENTAL RESULTS}

Confusion matrix that contains information about actual and predicted classifications done by a Support Vector Machine based on the query image are given below. We have experimented on 300 images of flower dataset containing three classes each of 100 images, which is divided into $50 \%-50 \%$ of training and testing database. Confusion matrix is used to represent the efficiency of distance measure depends upon number of returned images like for sunflower Mahalanobis Euclidean(L2), Spearman, Cityblock, Correlation gave good result, but for buttercup spearman gave good result but for Coneflower correlation distance measure gave good results. Precision indicates that an algorithm returned substantially more relevant results than irrelevant ones, while high recall means that an algorithm 
returned most of the relevant results. for spearman distance recall is $100 \%$, good for sunflower as shown in Fig8,Fig 9,Fig 10

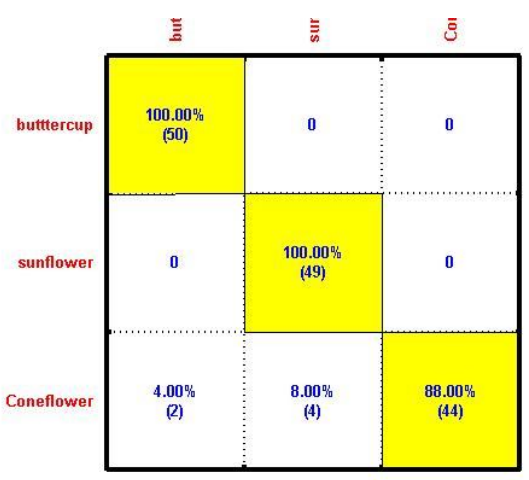

Figure 8 :Confusion Matrix when Distance Measure is Spearmen Distance

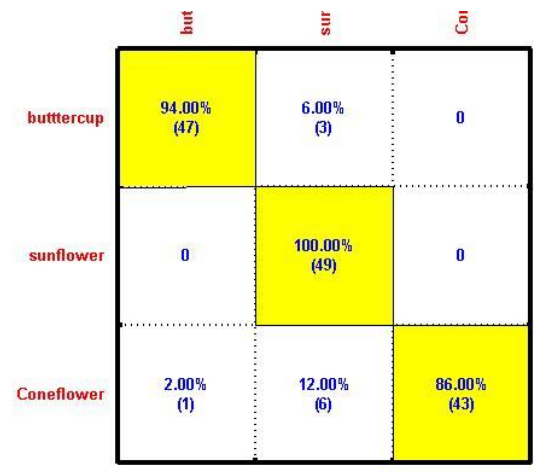

Figure 9: Confusion Matrix when Query image is Sunflower

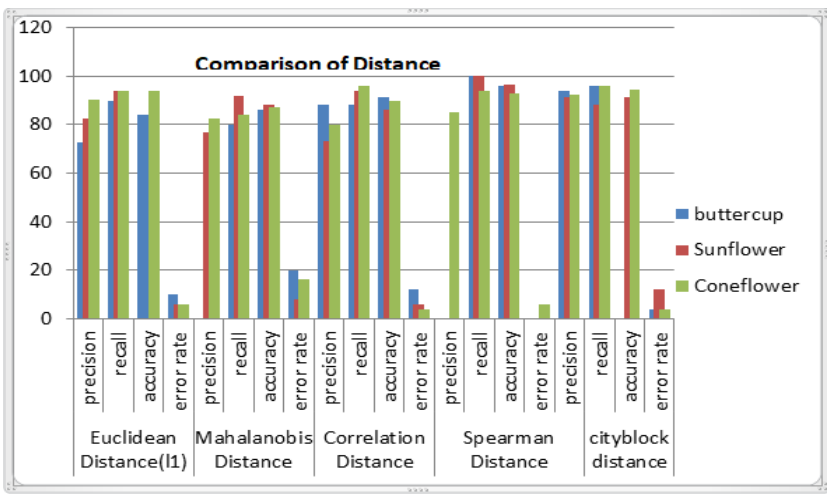

Figure 10: Comparsion for Mahalanobis, Euclidean, Correlation Cityblock, Spearman methods

\section{CONCLUSION}

The main contribution of our work is a method for selecting or retrieving the images is based on the query image as well distance measures used. Here we created a flower database of 300 images each consisting of 100 images of flowers of 3 different classes. Results are recorded for Mahalanobis, Distance, Euclidean Distance, Correlation Distance, City block, Spearman Distance. Efficiency of method depends on both distance method used as well as query image used for features extraction. When we given query image as a
Sunflower, Coneflower and buttercup flower of same color we used yellow flowers color, texture features is not only sufficient but also shape plays a prominent role the result obtained better for sunflower and when we gave a query image with different distance measure the result obtained better for Spearman algorithm. We also record accuracy of support vector machine for classification the spearman gives the better accuracy result for all the flowers.

\section{REFERENCES}

[1] Ruigang Fu, Biao Li, Yinghui Gao, Ping Wang ATR Key Lab ,Content-Based Image Retrieval Based on CNN and SVM 2016 2nd IEEE International Conference on Computer and Communications pg 638-642 https://doi.org/10.1109/CompComm.2016.7924779

[2] Mayank Jain and Divakar Singh Research Scholar India. A Survey on CBIR on the Basis of Different Feature Descriptor British Journal of Mathematics \& Computer Science 14(6): 1-13, 2016, Article no.BJMCS.24000 ISSN: 2231-0851 SCIENCEDOMAIN international https://doi.org/10.9734/BJMCS/2016/24000

[3] Ramesh K Lingadalli , N.Ramesh Content Based Image Retrieval using Color, Shape and Texture International Advanced Research Journal in Science, Engineering and Technology Vol. 2, Issue 6, June 2015 Copyright to IARJSET DOI 10.17148/IARJSET.2015.2610 40

[4] Satish Tunga, D.Jayadevappa \& C.Gururaj A Comparative Study of Content Based Image Retrieval Trends and Approaches International Journal of Image Processing (IJIP), Volume (9) : Issue (3) : 2015

[5] Nishant Shrivastava, Vipin Tyagi An efficient technique for retrieval of color images in large databases ,Computers and Electrical Engineering Volume 46 (2015) pg no 314-327 Elsevier https://doi.org/10.1016/j.compeleceng.2014.11.009

[6]Yogita Mistry, D.T. Ingole, M.D. Ingole Content based image retrieval using hybrid features and various distance metric Journal of Electrical Systems and Information Technology Volume 5 (2018) 874-888 Electronics Research Institute (ERI). Production and hosting by Elsevier

[7]P. Hiremath, and J. Pujari, "Content Based Image Retrieval using Color, Texture and Shape features," 15th international conference on advanced computing and communication, 2007.

[8]A.Ramesh Kumar, and D.Saravanan, Content Based Image Retrieval Using Color Histogram (IJCSIT) International Journal of Computer Science and Information Technologies, Vol. 4 (2), 2013.

[9] Recent Advance in Content-based Image Retrieval: A Literature Survey Wengang Zhou, Houqiang Li, and Qi Tian Fellow, IEEE 2 september 2017

[10]J.W.H. Tangelder and R.C. Veltkamp. A survey of content based 3D shape retrieval methods. Multimed Tools Appl, Springer Science + business Media LLc 2007 https://doi.org/10.1007/s11042-007-0181-0

[11]Piras, L., Giacinto, G.: Information fusion in content based image retrieval: a comprehensive overview. Inf. Fusion $37,50-60$ (2017) 
https://doi.org/10.1016/j.inffus.2017.01.003

[12] D. Sarala1, Tejas Kanikdaley , Sharad Jogi , Rahul K. Chaurasiya Content-Based Image Retrieval Using Hierarchical Color and Texture Similarity Calculation International Journal of Advanced Trends in Computer Science and Engineering(IJATCSE) Volume 7 No 2 March-April 2018

https://doi.org/10.30534/ijatcse/2018/02722018

[13] C. Jin and S. Ke. Content-Based Image Retrieval Based on Shape Similarity Calculation", 3D Research, vol. 8, no. 3, 2017.

https://doi.org/10.1007/s13319-017-0132-0

[14] G. Bindu Madhavi , V. Vijaya Kumar, K. Sasidhar A New Frame Work for Content Based Image Retrieval Based on Rule Based Motifs on Full Text on Images, International Journal of Advanced Trends in Computer Science and Engineering Volume 8 No. 4 July -August 2019 (IJATCSE) https://doi.org/10.30534/ijatcse/2019/15842019 